\title{
ARHANGEL'SKII SHEAF AMALGAMATIONS IN TOPOLOGICAL GROUPS
}

\author{
BOAZ TSABAN AND LYUBOMYR ZDOMSKYY
}

\begin{abstract}
We consider amalgamation properties of convergent sequences in topological groups and topological vector spaces. The main result of this paper is that, for arbitrary topological groups, Nyikos's property $\alpha_{1.5}$ is equivalent to Arhangel'ski1's formally stronger property $\alpha_{1}$. This result solves a problem of Shakhmatov (2002), and its proof uses a new perturbation argument. We also prove that there is a topological space $X$ such that the space $\mathrm{C}_{\mathrm{p}}(X)$ of continuous real-valued functions on $X$, with the topology of pointwise convergence, has Arhangel'ski1's property $\alpha_{1}$ but is not countably tight. This result follows from results of Arhangel'skiī-Pytkeev, Moore and Todorčević, and provides a new solution, with stronger properties than the earlier solution, of a problem of Averbukh and Smolyanov (1968) concerning topological vector spaces.
\end{abstract}

\section{Sheaf Amalgamations in topological GROUPS}

To avoid trivialities, by convergent sequence $x_{n} \rightarrow x$ we mean a proper one, that is, such that $x \neq x_{n}$ for all $n$. This way, convergence is a property of countably infinite sets: a countably infinite set $A$ converges to $x$ if all (equivalently, some) bijective enumerations of $A$ converge to $x$. Thus, in the following definition, by sequence we always mean a countably infinite set. The following concepts are due to Arhangel'skiı̌ [1, 2, except for $\alpha_{1.5}$ which is due to Nyikos [14].

Definition 1.1. A topological space $X$ is $\alpha_{i}$, for $i=1,1.5,2,3,4$, if, respectively, for each $x \in X$ and all pairwise disjoint sequences $S_{1}, S_{2}, \ldots \subseteq X$, each converging to $x$, there is a sequence $S \subseteq \bigcup_{n} S_{n}$ such that $S$ converges to $x$, and

$\left(\alpha_{1}\right) S_{n} \backslash S$ is finite for all $n$.

$\left(\alpha_{1.5}\right) S_{n} \backslash S$ is finite for infinitely many $n$.

$\left(\alpha_{2}\right) S_{n} \cap S$ is infinite for all $n$.

$\left(\alpha_{3}\right) S_{n} \cap S$ is infinite for infinitely many $n$.

$\left(\alpha_{4}\right) S_{n} \cap S$ is nonempty for infinitely many $n$.

1991 Mathematics Subject Classification. 26A03, 03E75.

Key words and phrases. Amalgamation of convergent sequences, $\alpha_{1}$ space, $\alpha_{1.5}$ space, L-space. 
A survey of these properties is available in [23]. In the integer-indexed properties $\alpha_{i}$, we may remove the requirement that the sequences $S_{1}, S_{2}, \ldots$ are pairwise disjoint [14]. Indeed, we can move to subsequences $S_{n}^{\prime}=S_{n} \backslash \bigcup_{k<n} S_{k}$ of $S_{n}$, for $n \in \mathbb{N}$. If $S_{n}^{\prime}$ is infinite for infinitely many $n$, we can dispose of the other ones. And if not, then the sequence $S:=\bigcup_{k<n} S_{k}$, for any $n$ with $S_{n}^{\prime}$ finite, would be as required in $\left(\alpha_{1}\right)$. However, removing the disjointness requirement in the property $\alpha_{1.5}$ renders it superfluous: Applying it to the modified sequence $\bigcup_{k \leq n} S_{k}$, for $n \in \mathbb{N}$, the obtained sequence $S$ would be as required in $\alpha_{1}$.

Each of the properties in Definition 1.1 implies the subsequent one. To see that $\alpha_{1.5}$ implies $\alpha_{2}$, for each $n$ decompose $S_{n}=\bigcup_{k} S_{n k}$, and take $S_{n}^{\prime}=\bigcup_{m \leq n} S_{m n}$ [14].

None of the implications

$$
\alpha_{1} \Rightarrow \alpha_{1.5} \Rightarrow \alpha_{2} \Rightarrow \alpha_{3} \Rightarrow \alpha_{4}
$$

can be (provably) reversed. Not even in the class of Fréchet-Urysohn spaces [23]. Recall that a topological space $X$ is Fréchet-Urysohn if each point in the closure of a set is in fact a limit of a sequence in that set.

In the present paper, we consider these properties in the context of topological groups. This direction was pioneered by Nyikos in his 1981 paper [13. In his paper, Nyikos proved that Fréchet-Urysohn groups are $\alpha_{4}$, and that sequential $\alpha_{2}$ groups are Fréchet-Urysohn. Shakhmatov [22] constructed, in the Cohen reals model, an example of a Fréchet-Urysohn group which is not $\alpha_{3}$, and a Fréchet-Urysohn $\alpha_{2}$ group which is not $\alpha_{1.5}$. In particular, none of the implications

$$
\alpha_{1} \Rightarrow \alpha_{2} \Rightarrow \alpha_{3} \Rightarrow \alpha_{4}
$$

is provably reversible in the realm of topological groups. The question whether $\alpha_{1.5}$ groups are $\alpha_{1}$ is implicit in Shakhmatov's paper. The problem whether Fréchet-Urysohn $\alpha_{1.5}$ groups are $\alpha_{1}$ is stated there. This variant of the problem was settled in the positive by Shibakov, in his 1999 paper [24].

In his 2002 chapter for Recent Progress in Topology [23], Shakhmatov cites Shibakov's solution, and writes: "It seems unclear if $\alpha_{1.5}$ and $\alpha_{1}$ are equivalent for all (i.e., not necessarily Fréchet-Urysohn) topological groups." For groups of the form $\mathrm{C}_{\mathrm{p}}(X)$, the continuous realvalued functions on a space $X$, with the topology of pointwise convergence, Sakai solved this problem in the positive [18]. One step in his solution, uses a pullback method which was used earlier by Scheepers [20] to show that for spaces of the form $\mathrm{C}_{\mathrm{p}}(X)$, we have that $\alpha_{2}=\alpha_{3}=\alpha_{4}$ : Replace the $n$-th sequence $\left\{f_{n m}: m \in \mathbb{N}\right\}$ by $\left\{\left|f_{1 m}\right|+\cdots+\left|f_{n m}\right|: m \in \mathbb{N}\right\}$. This approach is not applicable to arbitrary topological groups. Indeed, Sakai proves some of his lemmata in the context of general topological groups, but his main theorems are proved only in the case of $\mathrm{C}_{\mathrm{p}}(X)$. The following theorem answers Shakhmatov's question.

Theorem 1.2. A topological group is $\alpha_{1.5}$ if, and only if, it is $\alpha_{1}$. 
Proof. Let $G$ be a topological group, and $S_{1}, S_{2}, \ldots \subseteq G$ be sequences converging to $e$. Let $T$ be any sequence converging to $e$ (e.g., let $T:=S_{1}$ ). For each $n$, fix a bijective enumeration $S_{n}=\left\{g_{n m}: m \in \mathbb{N}\right\}$.

Let $\left\{\left(n_{k}, m_{k}\right): k \in \mathbb{N}\right\}$ be an enumeration of the set $\mathbb{N} \times \mathbb{N}$ where each pair $(n, m)$ appears infinitely often. For each $k$, as the set $\left(T \backslash\left\{t_{1}, \ldots, t_{k-1}\right\}\right) \cdot g_{n_{k} m_{k}}$ is infinite, we can pick an element

$$
t_{k} \in T \backslash\left\{t_{1}, \ldots, t_{k-1}\right\}
$$

such that

$$
t_{k} \cdot g_{n_{k} m_{k}} \notin\left\{t_{1} \cdot g_{n_{1} m_{1}}, \ldots, t_{k-1} \cdot g_{n_{k-1} m_{k-1}}\right\} .
$$

For each pair $(n, m)$, let $\{k(n, m, i): i \in \mathbb{N}\}$ be an increasing enumeration of the set $\left\{k:\left(n_{k}, m_{k}\right)=(n, m)\right\}$. Note that the function $(n, m, i) \mapsto k(n, m, i)$ is injective. For each $i$, define the following perturbation of $S_{n}$ :

$$
S_{n}^{(i)}=\left\{t_{k(n, 1, i)} \cdot g_{n 1}, t_{k(n, 2, i)} \cdot g_{n 2}, t_{k(n, 3, i)} \cdot g_{n 3}, \ldots\right\} .
$$

The sequence $S_{n}^{(i)}$ converges to $e$. By the construction, the sets $S_{n}^{(i)}$, for $n, i \in \mathbb{N}$, are pairwise disjoint, and therefore so are the sets

$$
S_{n}^{\prime}=S_{1}^{(n)} \cup S_{2}^{(n)} \cup \cdots \cup S_{n}^{(n)},
$$

for $n \in \mathbb{N}$. Being finite unions of sequences converging to $e$, the sequences $S_{1}^{\prime}, S_{2}^{\prime}, \ldots$ converge to $e$, too.

Apply $\alpha_{1.5}$ to the sequences $S_{1}^{\prime}, S_{2}^{\prime}, \ldots$, to find a sequence $S^{\prime}$ converging to $e$, such that the set $S_{n}^{\prime} \backslash S^{\prime}$ is finite for each $n$ in an infinite set $I \subseteq \mathbb{N}$. Define

$$
S:=\bigcup_{n \in I} \bigcup_{j=1}^{n}\left\{g_{j m}: m \in \mathbb{N}, t_{k(j, m, n)} \cdot g_{j m} \in S^{\prime}\right\} .
$$

Since for each $n \in I$ and each $j=1, \ldots, n$, we have that $t_{k(j, m, n)} \cdot g_{j m} \in S^{\prime}$ for all but finitely many $m$, the set $S_{j} \backslash S$ is finite for all $j$.

Finally, note that $S$ is obtained by taking a subsequence of $S^{\prime}$ and multiplying its elements by distinct elements $t_{k(j, m, n)}^{-1}$, that is elements of a subsequence of $\left\{t^{-1}: t \in T\right\}$, which also converges to $e$. Thus, $S$ converges to $e$, too.

We obtain a short proof of a result of Nogura and Shakhmatov.

Definition 1.3 (Nogura-Shakhmatov [12]). A topological space $X$ is Ramsey if, whenever $\lim _{n} \lim _{m} x_{n m}=x$, there is an infinite $I \subseteq \mathbb{N}$ such that for each neighborhood $U$ of $x$, there is $k$ such that $\left\{x_{n m}: k<n<m, n, m \in I\right\} \subseteq U$.

In general $\alpha_{1}$ topological spaces need not be Ramsey. In the context of topological groups, the above definition simplifies to the following one. 
Lemma 1.4 (Sakai [18]). A topological group $G$ is Ramsey if, and only if, whenever $\lim _{m} g_{n m}$ $=e$ for all $n$, there is an infinite set $I \subseteq \mathbb{N}$ such that the sequence $\left\{g_{n m}: n, m \in I, n<m\right\}$ converges to $e$.

Proof. Assume that $\lim _{m} g_{n m}=g_{n}$ and $\lim _{n} g_{n}=e$. For each $n$, define $g_{n m}^{\prime}=g_{n}^{-1} g_{n m}$. Then $\lim _{m} g_{n m}=e$ for all $n$.

Theorem 1.5 (Nogura-Shakhmatov [12]). Every $\alpha_{1.5}$ topological group is Ramsey.

Proof. Let $G$ be an $\alpha_{1.5}$ topological group. We establish the property stated in Lemma 1.4. Assume that $\lim _{m} g_{n m}=e$ for all $n$. By Theorem 1.2, $G$ is $\alpha_{1}$, and thus there is an increasing function $f: \mathbb{N} \rightarrow \mathbb{N}$ such that the sequence $\left\{g_{n m}: m \geq f(n)\right\}$ converges to $e$. Take $I$ to be the image of $f$.

\section{NeW Amalgamations}

Using the above-mentioned pullback method of Scheepers, Sakai proved that for groups of the form $\mathrm{C}_{\mathrm{p}}(X)$, Ramsey is equivalent to $\alpha_{2}$ [18]. The following problem, though, remains open.

Problem 2.1 (Shakhmatov [23]).

(1) Is every (Fréchet-Urysohn) $\alpha_{2}$ topological group Ramsey?

(2) Is every (Fréchet-Urysohn) Ramsey topological group $\alpha_{2}$ ?

In the forthcoming Definitions (2.2, 2.3, 2.5, and 2.7), we introduce several new local properties related to Ramsey and $\alpha_{2}$, and prove implications among them. The exact relations among these new properties and among them and the classic ones remain unknown. Some of the most interesting problems that remain open are summarized in Section 4 .

Definition 2.2. A topological space $X$ is locally Ramsey if, for each $x \in X$, whenever $\lim _{m} x_{n m}=x$ for all $n$, there is an infinite set $I \subseteq \mathbb{N}$ such that the sequence $\left\{x_{n m}: n, m \in\right.$ $I, n<m\}$ converges to $x$.

Locally Ramsey spaces are $\alpha_{3}$. By Lemma 1.4, a topological group is Ramsey if, and only if, it is locally Ramsey.

Definition 2.3. A topological space $X$ is $\alpha_{2^{-}}$if, for each $x \in X$, whenever $\lim _{m} x_{n m}=$ $x$ for all $n \in \mathbb{N}$, there are natural numbers $m_{1}<m_{2}<\cdots$ such that the sequence $\bigcup_{n}\left\{x_{1 m_{n}}, \ldots, x_{n m_{n}}\right\}$ converges to $x$.

Thus, every $\alpha_{2^{-}}$topological space is $\alpha_{2}$.

\section{Proposition 2.4.}

(1) Every $\alpha_{2-}$ topological space is locally Ramsey. 
(2) Every $\alpha_{2-}$ topological group is Ramsey.

Proof. (1) Take $m_{1}<m_{2}<\cdots$ as in the definition of $\alpha_{2^{-}}$, and set $I:=\left\{m_{n}: n \in \mathbb{N}\right\}$.

(2) By (1) and Lemma 1.4.

Definition 2.5. A topological space $X$ is $\alpha_{3^{-}}$if, for each $x \in X$, whenever $\lim _{m} x_{n m}=x$ for all $n$, there are infinite sets $I, J \subseteq \mathbb{N}$ such that the sequence $\left\{x_{n m}: n \in I, m \in J, n<m\right\}$ converges to $x$.

Thus, every locally Ramsey space is $\alpha_{3^{-}}$, and every $\alpha_{3^{-}}$space is $\alpha_{3}$. The above-mentioned results of Scheepers and Sakai follow.

Proposition 2.6. For topological groups of the form $\mathrm{C}_{\mathrm{p}}(X)$, the properties

$$
\alpha_{2^{-}}, \alpha_{2}, \alpha_{3^{-}}, \alpha_{3}, \alpha_{4} \text {, locally Ramsey, and Ramsey, }
$$

are equivalent.

Proof. By the above observations, it suffices to show that $\alpha_{4}$ implies $\alpha_{2^{-}}$for such spaces. This follows from Scheepers's pullback method: Given sequences $S_{n}=\left\{f_{n m}: m \in \mathbb{N}\right\}$, each converging to 0 , replace each sequence $S_{n}$ with

$$
S_{n}^{\prime}=\left\{\left|f_{1 m}\right|+\cdots+\left|f_{n m}\right|: m \geq n\right\} .
$$

Applying $\alpha_{4}$ and thinning out, we obtain an increasing sequence of indices $m_{1}<m_{2}<\cdots$ such that the sequence

$$
\left|f_{1 m_{n}}\right|+\cdots+\left|f_{n m_{n}}\right| \quad(n \in \mathbb{N})
$$

converges to 0 . Then the sequence $\bigcup_{n}\left\{f_{1 m_{n}}, \ldots, f_{n m_{n}}\right\}$ converges to 0 .

Definition 2.7. Let $X$ be a topological space, and $x \in X$. The game $\alpha_{2}^{\text {game }}(X, x)$ is played by two players, ONE and TWO, and has an inning per each natural number. On the $n$th inning, ONE chooses a sequence $S_{n}$ converging to $x$, and TWO responds by choosing a subsequence $T_{n} \subseteq S_{n}$. TWO wins if the sequence $\bigcup_{n} T_{n}$ converges to $x$. Otherwise, ONE wins.

Proposition 2.8. Assume that for each $x \in X$, ONE does not have a winning strategy in $\alpha_{2}^{\text {game }}(X, x)$. Then the space $X$ is $\alpha_{2^{-}}$(and thus locally Ramsey).

Proof. Assume that $\lim _{m} x_{n m}=x$ for all $n$. Consider the following strategy for ONE: In the first inning, ONE plays the sequence $\left\{x_{1 m}: m \in \mathbb{N}\right\}$. If TWO plays the subsequence

$$
\left\{x_{1 m}: m \in I_{1}\right\},
$$

then ONE responds by playing the sequence

$$
\left\{x_{2 m}: m \in I_{1} \backslash\left\{\min I_{1}\right\}\right\} .
$$


In general, if in the $n$th inning TWO chooses a subsequence

$$
\left\{x_{n m}: m \in I_{n}\right\},
$$

then ONE plays the sequence

$$
\left\{x_{n+1, m}: m \in I_{n} \backslash\left\{\min I_{n}\right\}\right\} .
$$

Since this strategy is not winning for ONE, there is a play lost by ONE. Let $I_{1}, I_{2}, \ldots$ be the infinite sets of sequence indices, which correspond to the moves of TWO in this play. Define $m_{n}:=\min I_{n}$ for each $n$. Then, for each $n$,

$$
\bigcup_{n \in \mathbb{N}}\left\{x_{1 m_{n}}, \ldots, x_{n m_{n}}\right\} \subseteq \bigcup_{n \in \mathbb{N}} T_{n},
$$

and the latter sequence converges to $x$.

Corollary 2.9. Let $G$ be a topological group. If ONE does not have a winning strategy in the game $\alpha_{2}^{\text {game }}(G, e)$, then $G$ is Ramsey (indeed, $\alpha_{2^{-}}$).

Proposition 2.10. Let $X$ be an $\alpha_{1}$ space. For each $x \in X$, ONE does not have a winning strategy in the game $\alpha_{2}^{\text {game }}(X, x)$.

Proof. Define the game $\alpha_{1}^{\text {game }}(X, x)$ corresponding to the property $\alpha_{1}$ (at $\left.x\right)$. This game is similar to $\alpha_{2}^{\text {game }}(X, x)$, with the only difference that here, TWO must choose a cofinite subset of each sequence provided by ONE.

Lemma 2.11. A topological space $X$ is $\alpha_{1}$ if, and only if, for each point $x \in X, O N E$ does not have a winning strategy in the game $\alpha_{1}^{\text {game }}(X, x)$.

Proof. $(\Leftarrow)$ Immediate.

$(\Rightarrow)$ The following method was used by Scheepers in [19] to prove similar results for games involving open covers.

Fix a strategy for ONE in $\alpha_{1}^{\text {game }}(X, x)$. For each sequence played by ONE, there are only countably many possible legal responds by TWO. Let $\mathcal{F}$ be the family of all possible sequences which ONE may play according to the fixed strategy. As the family $\mathcal{F}$ is countable, we can apply $\alpha_{1}$ to $\mathcal{F}$, and find for each sequence $S \in \mathcal{F}$ a cofinite subset $S^{\prime} \subseteq S$, such that the sequence $\bigcup_{S \in \mathcal{F}} S^{\prime}$ converges to $x$.

Consider a play where TWO responds to each given sequence $S_{n}$ by the sequence $S_{n}^{\prime}$. This play is lost by ONE.

If ONE does not have a winning strategy in $\alpha_{1}^{\text {game }}(X, x)$, then ONE does not have one in $\alpha_{2}^{\text {game }}(X, x)$, where the moves of TWO are less restricted. 


\section{Sheaf amalgamations in topological Vector spaces}

In their 1968 paper [6], Averbukh and Smolyanov asked whether every $\alpha_{1}$ topological vector space is Fréchet-Urysohn. The problem was only settled in Plichko's 2008 paper [16], using Banach spaces with certain weak topologies. Knowledge that was available in the field of selection principles, even before its solidification in 1996 [19, 9], was enough to have a consistent counterexample for the Averbukh-Smolyanov problem: Assume the Continuum Hypothesis, and let $S \subseteq \mathbb{R}$ be a Sierpiński set, that is, a set of size continuum whose intersection with every Lebesgue-null set is countable. It is known that every Borel image of a Sierpiński set in $\mathbb{N}^{\mathbb{N}}$ is bounded, and consequently the space $\mathrm{C}_{\mathrm{p}}(S)$ is $\alpha_{1}$. On the other hand, the space $\mathrm{C}_{\mathrm{p}}(S)$ cannot be Fréchet-Urysohn since the set $S$ is not Lebesgue-null [8]. Moreover, there is an example based solely on cardinality: It is known that the combinatorial cardinal $\mathfrak{p}$ (respectively, $\mathfrak{b}$ ) is the minimal cardinality of a set $X \subseteq \mathbb{R}$ such that the space $\mathrm{C}_{\mathrm{p}}(X)$ is not Fréchet-Urysohn (respectively, $\alpha_{1}$ ). Thus, the consistent assumption $\mathfrak{p}<\mathfrak{b}$ provides a counterexample in a trivial manner. We show in Theorem 3.3 that this approach provides a counterexample, within ZFC. Moreover, this example has the following remarkable properties: Every separable subspace is metrizable, but the topological vector space is not even countably tight.

In the proof of Theorem 3.3 , we will use several known facts, for which we provide proofs for completeness.

General versions of the following fact were proved in the 1970's (e.g., [10] and references therein). Recall that the $\Sigma$-product of spaces $X_{i}$, for $i \in I$, with respect to a point $x \in$ $\prod_{i \in I} X_{i}$, is the subspace $\Sigma_{i \in I} X_{i}$ of the product space $\prod_{i \in I} X_{i}$, consisting of all $y \in \prod_{i \in I} X_{i}$ such that $y_{i}=x_{i}$ for all but countably many $i \in I$.

Proposition 3.1. Let $X$ be a $\Sigma$-product of a family of first countable spaces. Then:

(1) Every countable subspace of $X$ is first countable.

(2) The space $X$ is $\alpha_{1}$.

(3) The space $X$ has countable tightness.

(4) The space $X$ is Fréchet-Urysohn.

Proof. (1) Countable subspaces of $X$ are supported on a countable set of indices.

(2) follows from (1).

(3) Let $X=\Sigma_{i \in I} X_{i}, A \subseteq X$ and $y \in \bar{A}$. For each $i \in I$, let $\mathcal{B}_{i}$ be a countable base at $y_{i}$. For a finite set $F \subseteq I$ and an element $U \in \prod_{i \in F} \mathcal{B}_{i}$, let

$$
[U]:=\left\{x \in X: \forall i \in F, x_{i} \in U_{i}\right\} .
$$

Fix an arbitrary, countably infinite set $I_{1} \subseteq I$. Continue by induction on $n$. Let $A_{n} \subseteq A$ be a countable set intersecting $[U]$ for all finite $F \subseteq I_{n}$ and all $U \in \prod_{i \in F} \mathcal{B}_{i}$. Let $I_{n+1}$ be the union of $I_{n}$ and the supports of the elements of $A_{n}$. 
The point $y$ is in the closure of the countable set $\bigcup_{n} A_{n}$. Indeed, let $F$ be a finite subset of $I$, and $U \in \prod_{i \in F} \mathcal{B}_{i}$. Let $F_{1}=F \cap \bigcup_{n} I_{n}$, and $F_{2}=F \backslash \bigcup_{n} I_{n}$. As the set $F$ is finite, there is $n$ such that $F_{1} \subseteq I_{n}$. Let $V=\left(U_{i}: i \in F_{1}\right)$. Then there is an element $a \in A_{n} \cap[V]$. As the support of $a$ is contained in $I_{n+1}, a_{i}=y_{i}$ for all $i \in F_{2}$. Thus, $a \in[U]$.

(4) follows from (3) and (1).

The following result, brought to our attention by J. Moore, is proved for S in [27, Theorem 7.10], where it is pointed out that the $\mathrm{L}$ case is analogous. For completeness, we provide a proof for the $\mathrm{L}$ case, which is the one needed here.

Lemma 3.2. Assume that $Y$ is a regular topological space with all finite powers Lindelöf and countably tight, and $X$ is a non-separable subspace of $Y$. There exists a c.c.c. poset $\mathbb{P}$ such that, in $V^{\mathbb{P}}$, the space $X$ has an uncountable discrete subspace.

Proof. It suffices to show that there are a c.c.c. poset $\mathbb{P}$ and a family $\mathcal{D}$ of $\aleph_{1}$ many dense subsets of $\mathbb{P}$ such that:

For each ZFC model $V^{\prime} \supseteq V$ with $\omega_{1}^{V^{\prime}}=\omega_{1}^{V}$, if there is in $V^{\prime}$ a filter $G \subseteq \mathbb{P}$ meeting each $D \in \mathcal{D}$, then the space $X$ has an uncountable discrete subspace in $V^{\prime}$.

Passing to a subset of $X$, if necessary, we may assume that $X=\left\{x_{\xi}: \xi<\omega_{1}\right\}$ and ${\overline{\left\{x_{\xi}: \xi<\alpha\right\}}}^{Y} \cap\left\{x_{\eta}: \eta \geq \alpha\right\}=\emptyset$ for every $\alpha<\omega_{1}$. There are two cases to consider.

Case 1. ${\overline{\left\{x_{\xi}: \xi<\alpha\right\}}}^{Y} \cap{\overline{\left\{x_{\eta}: \eta \geq \alpha\right\}}}^{Y}=\emptyset$ for all $\alpha<\omega_{1}$; in other words, $X$ is a free sequence in $Y$. Since the space $Y$ has countable tightness, $\bar{X}^{Y}=\bigcup_{\alpha<\omega_{1}}{\overline{\left\{x_{\beta}: \beta<\alpha\right\}}}^{Y}$. The space $\bar{X}^{Y}$ is closed in $Y$, and thus Lindelöf. On the other hand, the family

$$
\left\{\bar{X}^{Y} \backslash{\overline{\left\{x_{\beta}: \beta \geq \alpha\right\}}}^{Y}: \alpha<\omega_{1}\right\}
$$

is an open cover of $\bar{X}^{Y}$ without a countable subcover; a contradiction.

Case 2. ${\overline{\left\{x_{\xi}: \xi<\alpha\right\}}}^{Y} \cap{\overline{\left\{x_{\eta}: \eta \geq \alpha\right\}}}^{Y} \neq \emptyset$ for some $\alpha$. In particular, the set $\left\{x_{\eta}: \eta \geq \alpha\right\}$ is not compact. We may assume that the space $X$ is not compact. Let $\mathcal{U}$ be an ultrafilter on $X$ whose elements are uncountable. If there exists some $\alpha$ such that $\mathcal{U}$ contains all open neighborhoods in $X$ of $x_{\alpha}$, then the Hausdorff property implies that every $x_{\beta}$ for $\beta \neq \alpha$ has a neighborhood in $X$ which is not in $\mathcal{U}$. By removing a point from $X$, if needed, we may assume that every element of $X$ has a neighborhood in $X$ that is not in $\mathcal{U}$.

For each $\alpha$, pick neighborhoods $U_{\alpha}, V_{\alpha}$ of $x_{\alpha}$ in $Y$ such that $\overline{V_{\alpha}} \subseteq U_{\alpha}, \overline{U_{\alpha}} \cap \overline{\left\{x_{\xi}: \xi<\alpha\right\}}=$ $\emptyset$, and $\left\{U_{\alpha} \cap X: \alpha<\omega_{1}\right\} \subseteq P(X) \backslash \mathcal{U}$. Then finitely many sets $U_{\alpha}$ cannot cover a cocountable subset of $X$. Let $\mathbb{P}$ be the poset consisting of all finite sets $\left\{\alpha_{0}, \ldots, \alpha_{n-1}\right\} \subseteq \omega_{1}$, 
$\alpha_{0}<\cdots<\alpha_{n-1}$, such that $x_{\alpha_{j}} \notin V_{\alpha_{i}}$ whenever $i<j$. A condition $H$ is stronger than $F$, $H \leq F$, if $F \subseteq H$

Assume, towards a contradiction, that there is an uncountable antichain $\left\{F_{\alpha}: \alpha<\omega_{1}\right\}$ in $\mathbb{P}$. For incompatible elements $F, H \in \mathbb{P}$, the elements $F \backslash H$ and $H \backslash F$ are also incompatible. By the $\Delta$-System Lemma, we may assume that the sets $F_{\alpha}$ are pairwise disjoint, $\min F_{\alpha}>$ $\max F_{\beta}$ for all $\beta<\alpha$, and $\left|F_{\alpha}\right|=n$ for all $\alpha$. For each $\alpha$, let $\left\{\xi_{\alpha}^{0}, \ldots, \xi_{\alpha}^{n-1}\right\}$ be the increasing enumeration of $F_{\alpha}$. Set

$$
\begin{aligned}
W_{\alpha}^{0} & :=\left\{\left(x_{0}, \ldots, x_{n-1}\right) \in X^{n}: \forall i, j<n,\left(x_{i} \notin U_{\xi_{\alpha}^{j}}\right)\right\}, \\
W_{\alpha}^{1} & :=\left\{\left(x_{0}, \ldots, x_{n-1}\right) \in X^{n}: \exists i, j<n,\left(x_{i} \in \overline{V_{\xi_{\alpha}^{j}}}\right)\right\} .
\end{aligned}
$$

Then $W_{\alpha}^{0} \cap W_{\alpha}^{1}=\emptyset$, and the sets $W_{\alpha}^{0}, W_{\alpha}^{1}$ are closed. Moreover, by our choice of the sets $U_{\delta}$, we have that $\left(x_{\xi_{\beta}^{i}}\right)_{i<n} \in W_{\alpha}^{0}$ for all $\beta<\alpha$. By the definition of $\mathbb{P}$ and the incompatibility of the sets $F_{\alpha}$ and $F_{\beta}$, we have that $\left(x_{\xi_{\beta}^{i}}\right)_{i<n} \in W_{\alpha}^{1}$ for all $\beta>\alpha$. Thus, the subset $A:=\left\{\vec{x}_{\alpha}:=\left(x_{\xi_{\alpha}^{i}}\right)_{i<n}: \alpha<\omega_{1}\right\}$ of $X^{n}$ satisfies

$$
{\overline{\left\{\vec{x}_{\beta}: \beta<\alpha\right\}}}^{Y^{n}} \cap{\overline{\left\{\vec{x}_{\beta}: \beta \geq \alpha\right\}}}^{Y^{n}}=\emptyset
$$

for all $\alpha$; a contradiction.

Thus, the forcing notion $\mathbb{P}$ is c.c.c. For each $\alpha<\omega_{1}$, let $D_{\alpha}:=\{F \in \mathbb{P}: \max F>\alpha\}$. Since no finite subfamily of $\left\{U_{\alpha}: \alpha<\omega_{1}\right\}$ covers a co-countable subset of $X$, each set $D_{\alpha}$ is dense in $\mathbb{P}$. Assume that $G$ is a subfilter of $\mathbb{P}$ (possibly, in some extension $V^{\prime} \supseteq V$ ) which intersects every set $D_{\alpha}$. Then $x_{\beta} \notin V_{\alpha}$ for all $\beta, \alpha \in \bigcup G$ : if $\beta<\alpha$ this follows from the choice of $V_{\alpha}$, and if $\beta>\alpha$ this follows from the existence of an element $F \in G$ containing both $\alpha$ and $\beta$. Thus, $G$ gives rise to the discrete subspace $\left\{x_{\alpha}: \alpha \in \bigcup G\right\}$ of $X$, which is uncountable if $\omega_{1}^{V^{\prime}}=\omega_{1}^{V}$.

We are ready for the main result of this section. An $L$-space is a hereditarily Lindelöf nonseparable topological space. The existence of L-spaces was established by Moore in [11]. A classical result of Arhangel'skil and, independently, Pytkeev, asserts that a function space $\mathrm{C}_{\mathrm{p}}(X)$ has countable tightness if and only if all finite powers of the space $X$ are Lindelöf.

Theorem 3.3. There is a hereditarily Lindelöf nonseparable Fréchet-Urysohn space L, such that:

(1) The space $\mathrm{C}_{\mathrm{p}}(L)$ is $\alpha_{1}$; moreover, every separable subspace of $\mathrm{C}_{\mathrm{p}}(L)$ is metrizable.

(2) The space $\mathrm{C}_{\mathrm{p}}(L)$ is not Fréchet-Urysohn; moreover, it is not countably tight.

Proof. Let $L$ be an L-space of the kind constructed by Moore [11. Following Todorčević [26], Moore considered a function osc: $\left\{(\alpha, \beta) \in \omega_{1}^{2}: \alpha<\beta\right\} \rightarrow \omega$ with strong combinatorial properties. Let $\left(z_{\alpha}\right)_{\alpha<\omega_{1}}$ be a sequence of rationally independent points on the multiplicative 
circle group $\mathbb{T}=\{z \in \mathbb{C}:|z|=1\}$. For each $\beta<\omega_{1}$, define an element $w_{\beta} \in \mathbb{T}^{\omega_{1}}$ by

$$
w_{\beta}(\alpha)= \begin{cases}z_{\alpha}^{\mathrm{osc}(\alpha, \beta)+1} & \alpha<\beta \\ 1 & \text { otherwise }\end{cases}
$$

By Theorem 7.11 of [11], the set $L=\left\{w_{\beta}: \beta<\omega_{1}\right\}$ is an L-space. By Theorem 7.8 of [11], or directly by Proposition 3.1 , the space $L$ is Fréchet-Urysohn.

(1) Let $D$ be a countable subset of $\mathrm{C}_{\mathrm{p}}(L)$. Since $L$ is a hereditarily Lindelöf subspace of a product space and $\mathbb{R}$ is a second countable Hausdorff space, every continuous function $f: L \rightarrow \mathbb{R}$ is determined by countably many coordinates; equivalently, there are $\alpha<\omega_{1}$ and a continuous function $g_{\alpha}: \operatorname{pr}_{\alpha}[L] \rightarrow \mathbb{R}$ such that $f=g_{\alpha} \circ \operatorname{pr}_{\alpha}$.

Lemma 3.4. For each $\alpha<\omega_{1}$, the set $\operatorname{pr}_{\alpha}[L]$ is countable.

Proof. By [11, Proposition 7.13], the subtree $\{\operatorname{osc}(\cdot, \delta) \uparrow \alpha: \delta \geq \alpha\}$ of $\omega^{<\omega_{1}}$ is Aronszajn, where $\operatorname{osc}(\cdot, \delta): \xi \mapsto \operatorname{osc}(\xi, \delta)$ for $\xi<\delta$. By the definition of Aronszajn tree, the set

$$
\left\{\operatorname{osc}(\cdot, \delta) \uparrow \alpha: \alpha<\delta<\omega_{1}\right\}
$$

is countable for each $\alpha<\omega_{1}$. Thus, the set $\left\{w_{\delta}\left\lceil\alpha: \alpha<\delta<\omega_{1}\right\}\right.$ is countable, and hence so is the set $\operatorname{pr}_{\alpha}[L]$.

As the set $D$ is countable, there is $\alpha<\omega_{1}$ such that every function $f \in D$ is determined by a continuous function on the first $\alpha$ coordinates. Thus, the function

$$
\begin{aligned}
\operatorname{pr}_{\alpha}^{*}: \mathrm{C}_{\mathrm{p}}\left(\operatorname{pr}_{\alpha}[L]\right) & \rightarrow \mathrm{C}_{\mathrm{p}}(L) \\
g & \mapsto g \circ \mathrm{pr}_{\alpha}
\end{aligned}
$$

is an embedding (e.g., [3, Proposition 0.4.6]). As the set $\operatorname{pr}_{\alpha}[L]$ is countable, the space $\mathrm{C}_{\mathrm{p}}\left(\operatorname{pr}_{\alpha}[L]\right)$ is metrizable, and therefore so is its image, which contains $D$.

(2) By Lemma 3.1, every finite power of $\Sigma_{\alpha<\omega_{1}} \mathbb{T}$ is countably tight. As countable tightness is hereditary, all finite powers of $L$ are countably tight. By Lemma 3.2 with $X=Y=L$, we have that if all finite powers of $L$ are Lindelöf, then there is a c.c.c. poset $\mathbb{P}$ such that $L$ has an uncountable discrete subspace in $V^{\mathbb{P}}$. But in the proof of [11, Theorem 7.17], it is pointed out that the space $L$ remains an L-space in c.c.c. forcing extensions. In fact, c.c.c. is not necessary, as the following lemma shows.

Lemma 3.5. Moore's L-space remains an L-space in every forcing extension that does not collapse $\aleph_{1}$.

Proof sketch. In accordance with [11, Definition 2.1], the construction of $L$ is based on a $C$-sequence

$$
\bar{C}=\left\langle C_{\alpha}: \alpha<\omega_{1}, \alpha \text { limit }\right\rangle
$$


The function osc is constructed from $\bar{C}$ in a way that, for each poset $\mathbb{P}$ preserving $\omega_{1}$, the constructions of osc in $V$ and in $V^{\mathbb{P}}$ give the same function, and hence give rise to the same subspace of the $\Sigma$ product of circles 1 - By the same proof carried out in $V^{\mathbb{P}}$, this space is an $L$ space in $V^{\mathbb{P}}$.

It follows that some finite power of $L$ is not Lindelöf. By the Arhangel'skiu-Pytkeev Theorem, the space $\mathrm{C}_{\mathrm{p}}(X)$ is not countably tight.

\section{Open PROBLEMS AND CLOSING REMARKS}

By Section 2 we have that, for topological groups,

$$
\alpha_{1} \Leftrightarrow \alpha_{1.5} \Rightarrow \operatorname{ONE} \uparrow \alpha_{2}^{\text {game }}(G, e) \Rightarrow \alpha_{2^{-}} \Rightarrow \text { Ramsey } \Rightarrow \alpha_{3^{-}} \Rightarrow \alpha_{3} \Rightarrow \alpha_{4}
$$

and $\alpha_{2^{-}} \Rightarrow \alpha_{2} \Rightarrow \alpha_{3}$.

Problem 4.1. Are there, in ZFC or consistently, topological groups $G$ that are

(1) $\alpha_{3}$ but not $\alpha_{3^{-}}$?

(2) $\alpha_{3^{-}}$but not Ramsey?

(3) Ramsey but not $\alpha_{2^{-}}$?

(4) $\alpha_{2}$ - but ONE has a winning strategy in $\alpha_{2}^{\text {game }}(G, e)$ ?

(5) not $\alpha_{1}$ and ONE has no winning strategy in $\alpha_{2}^{\text {game }}(G, e)$ ?

(6) $\alpha_{2}$ but not $\alpha_{2^{-}}$?

(7) $\alpha_{3^{-}}$but not $\alpha_{2}$ ?

Problem 4.2. Let $X$ be a Tychonoff space such that the function space $\mathrm{C}_{\mathrm{p}}(X)$ satisfies $\alpha_{2}$. Does it follow that $O N E$ does not have a winning strategy in the game $\alpha_{2}^{\text {game }}\left(\mathrm{C}_{\mathrm{p}}(X), 0\right)$ ?

The results and methods of Section 3 are already used in a number of papers, including [4, 5, 15, 21, 25]. The direct union of an $L$-space and the Sorgenfrey line is an $L$-space with non-Lindelöf square. However, such a space does not enjoy the properties described in Section 3. Answering a question from an earlier version of this paper, Yinhe Peng proved that the square of Moore's original L-space is also non-Lindelöf [15]. Peng's arguments are highly nontrivial, and use the fine details of Moore's construction. Our proof in item 2 of Theorem 3.3 is potentially more general, as it applies to all absolute modifications of Moore's construction where Lemma 3.5 holds. We do not know any modification of Moore's construction where Lemma 3.5 fails.

\footnotetext{
${ }^{1}$ This can be checked by going through the relevant definitions in [11, $\S 4$ ], without involving any deep absoluteness arguments.
} 
Acknowledgments. We thank Vaja Tarieledze for bringing relevant bibliography to our attention and for vivid discussions. We thank Franklin Tall for comments which helped to improve the presentation of the second part of the paper. We also thank Assaf Rinot for an interesting discussion regarding Lemma 3.5. We are indebted to Justin Moore for bringing Lemma 3.2 to our attention, and to the referee for his time and effort. The second named author was supported by the FWF grant I 1209-N25 and by the Austrian Academy of Sciences, through the APART Program. A part of this work was carried out while the first named author was on Sabbatical leave at the Weizmann Institute of Science. This author thanks his hosts for their kind hospitality.

\section{REFERENCES}

[1] A. Arhangel'skiŭ, The frequency spectrum of a topological space and the classification of spaces, Soviet Mathematical Doklady 13 (1972), 1186-1189.

[2] A. Arhangel'skiu, The frequency spectrum of a topological space and the product operation, Translations of the Moscow Mathematical Society 2 (1981) 163-200.

[3] A. Arhangel'skiॅ, Topological Function Spaces, Kluwer Academic Publishers, 1992.

[4] L. Aurichi, R. Dias, Topological games and Alster spaces, Canadian Mathematical Bulletin 57 (2014), 683-696.

[5] L. Aurichi, F. Tall, Lindelöf spaces which are indestructible, productive, or D, Topology and its Applications 159 (2012), 331-340.

[6] V. Averbukh, O. Smolyanov, The various definitions of the derivative in linear topological spaces, Russian Mathematical Surveys 23 (1968), 67-113.

[7] H. Corson, Normality in subsets of product spaces, American Journal of Mathematics 81 (1959), 785796.

[8] J. Gerlits, Zs. Nagy, Some properties of $C(X)$, I, Topology and its Applications 14 (1982), 151-161.

[9] W. Just, A. Miller, M. Scheepers, P. Szeptycki, The combinatorics of open covers II, Topology and its Applications 73 (1996), 241-266.

[10] A. Kombarov, The tightness and normality of $\Sigma$-products, Soviet Mathematical Doklady 239 (1978), $775-778$.

[11] J. Moore, A solution to the L-space problem, Journal of the American Mathematical Society 19 (2006), $717-736$.

[12] T. Nogura, D. Shakhmatov, Amalgamation of convergent sequences in locally compact groups, Comptes Rendus Mathematique 320, 1349-1354.

[13] P. Nyikos, Metrizability and the Fréchet-Urysohn property in topological groups, Proceedings of the American Mathematical Society 83 (1981), 793-801.

[14] P. Nyikos, Subsets of $\omega^{\omega}$ and the Fréchet-Urysohn and $\alpha_{i}$-properties, Topology and its Applications 48 (1992), 91-116.

[15] Y. Peng, An L space with non-Lindelöf square, Topology Proceedings 46 (2015), 233-242.

[16] A. Plichko, Diagonal sequence property in Banach spaces with weaker topologies, Journal of Mathematical Analysis and Applications 350 (2009), 838-844.

[17] D. Repovš, L. Zdomskyy, A new Lindelöf topological group, Topology and its Applications 157 (2010), 990-996.

[18] M. Sakai, The Ramsey property for $\mathrm{C}_{\mathrm{p}}(X)$, Acta Mathematica Hungarica 128 (2010), 96-105. 
[19] M. Scheepers, Combinatorics of open covers I: Ramsey theory, Topology and its Applications 69 (1996), $31-62$.

[20] M. Scheepers, $\mathrm{C}_{\mathrm{p}}(X)$ and Arhangel'skiu's $\alpha_{i}$ spaces, Topology and its Applications 89 (1998), 265-275.

[21] M. Scheepers, F. Tall, Errata to "Lindelöf indestructibility, topological games and selection principles", Fundamenta Mathematicae 224 (2014), 203-204.

[22] D. Shakhmatov, $\alpha_{i}$-properties in Fréchet-Urysohn topological groups, Topology Proceedings 15 (1990), 143-183.

[23] D. Shakhmatov, Convergence in the presence of algebraic structure, in: Recent progress in general topology, II, North-Holland, Amsterdam, 2002, 463-484.

[24] A. Shibakov, Countable Fréchet topological groups under CH, Topology and its Applications 91 (1999), 119-139.

[25] F. Tall, Productively Lindelof spaces may all be D, Canadian Mathematical Bulletin 56 (2013), 203-212.

[26] S. Todorčević, Partitioning pairs of countable ordinals, Acta Mathematica 159 (1989), 261-294.

[27] S. Todorčević., Partition problems in topology, Contemporary Mathematics 84, American Mathematical Society, Providence, RI, 1989.

[28] B. Tsaban, Strong $\gamma$-sets and other singular spaces, Topology and its Applications 153 (2005), 620-639.

[29] B. Tsaban, L. Zdomskyy, Hereditarily Hurewicz spaces and Arhangel'skiu sheaf amalgamations, Journal of the European Mathematical Society 12 (2012), 353-372.

(Tsaban) Department of Mathematics, Bar-Ilan University, Ramat Gan 5290002, Israel and Department of Mathematics, Weizmann Institute of Science, Rehovot 7610001, Israel

E-mail address: tsaban@math.biu.ac.il

URL: http://math.biu.ac.il/ ${ }^{\text {tsaban }}$

(Zdomskyy) Kurt Gödel Research Center for Mathematical Logic, University of Vienna, WÄhringer Str. 25, 1090 Vienna, Austria

E-mail address: lzdomsky@logic.univie.ac.at

$U R L:$ http://www.logic.univie.ac.at/ 1 ldomsky 